\title{
REITERATED HOMOGENIZATION OF DEGENERATE NONLINEAR ELLIPTIC EQUATIONS
}

\author{
J. BYSTRÖM* J. ENGSTRÖM* P. WALL* \\ (Dedicated to the memory of Jacques-Louis Lions)
}

\begin{abstract}
The authors study homogenization of some nonlinear partial differential equations of the form $-\operatorname{div}\left(a\left(h x, h^{2} x, D u_{h}\right)\right)=f$, where $a$ is periodic in the first two arguments and monotone in the third. In particular the case where $a$ satisfies degenerated structure conditions is studied. It is proved that $u_{h}$ converges weakly in $W_{0}^{1,1}(\Omega)$ to the unique solution of a limit problem as $h \rightarrow \infty$. Moreover, explicit expressions for the limit problem are obtained.
\end{abstract}

Keywords Homogenization, Reiterated, Monotone, Degenerated

2000 MR Subject Classification 35B27, 35J70, 74Q99

Chinese Library Classification O175.21, O175.25 Document Code A

Article ID 0252-9599(2002)03-03251-10

\section{$\S 1$. Introduction}

This paper is devoted to homogenization of partial differential operators including several periodically oscillating length scales. This type of equations appear in many fields of physics and engineering sciences where the physical phenomena occur in highly heterogeneous media. One example is heat conduction in composite materials involving two different materials which are periodically distributed. The local characteristics are then described by rapidly oscillating functions. A direct numerical treatment of such problems is often impossible due to the rapidly oscillating functions and one has to apply some type of asymptotic analysis. The branch of mathematics developed for the analysis of these types of problems is known as homogenization. For more information concerning the homogenization theory, the reader is referred to $[1,2,7,10]$ and $[12]$.

We will now give a short overview of previous results connected to this work and explain what our contribution is. Let us consider the class of partial differential equations of the form

$$
-\operatorname{div}\left(a_{h}\left(x, D u_{h}\right)\right)=f \quad \text { on } \Omega, u_{h} \in W_{0}^{1, p}(\Omega),
$$

where $a_{h}$ is increasingly oscillating as $h \rightarrow \infty, \Omega$ is an open bounded subset of $\mathbf{R}^{n}, 1<p<$ $\infty, 1 / p+1 / q=1$ and $f \in W^{-1, q}(\Omega)$. The homogenization problem for (1.1) consists of the study of the asymptotic behavior of solutions $u_{h}$ as $h \rightarrow \infty$. In many important cases $u_{h}$ converges weakly in $W_{0}^{1, p}(\Omega)$ to the solution $u$ of the homogenized problem

$$
-\operatorname{div}\left(b(D u)=f \quad \text { on } \Omega, u \in W_{0}^{1, p}(\Omega) .\right.
$$

In [6] and [11] the following situation was studied: $a_{h}$ is of the form $a_{h}(x, \xi)=a(h x, \xi)$, where $a$ is monotone, continuous and satisfies suitable coerciveness and growth conditions in the second argument and is periodic in the first argument. A corresponding homogenization

\footnotetext{
Manuscript received January 31, 2002.

*Department of Mathematics, Luleå University of Technology, SE-971 87 Luleå, Sweden.

E-mail: wall@sm.luth.se
} 
result, with the difference that $a$ only satisfies degenerate structure conditions, was obtained in [9]. In this situation it is natural to work with weighted spaces which means that instead of (1.1) we have

$$
-\operatorname{div}\left(a\left(h x, D u_{h}\right)\right)=f \quad \text { on } \Omega, u_{h} \in W_{0}^{1, p}\left(\Omega, \lambda_{h}\right),
$$

where $\left(\lambda_{h}\right)$ is a sequence of periodic weights.

In the case when $a_{h}(x, \xi)=a\left(h x, h^{2} x, \xi\right)$, where $a$ is periodic in the first two variables, one speaks about reiterated homogenization. This concept was introduced by Bensoussan, Lions and Papanicolaou in [1], where it was stated a result for linear operators. Concerning reiterated homogenization of nonlinear problems we refer to [13] and [14]. One important application of reiterated homogenization is that it has been an indispensable tool in the construction of structures with extreme effective material properties. Concerning this topic we refer to the collection of classical papers in [5], where the introduction gives a good selection of references. We remark that some of the homogenization problems above also have been studied by $\Gamma$-convergence for the corresponding variational problems and by twoscale convergence, but leave out this discussion since it is out of the scope of this work.

In this paper we study reiterated homogenization where $a$ only satisfies degenerate structure conditions. More precisely we prove that the solutions $u_{h}$ of

$$
-\operatorname{div}\left(a\left(h x, h^{2} x, D u_{h}\right)\right)=f \quad \text { on } \Omega, u_{h} \in W_{0}^{1, p}\left(\Omega, \lambda_{h}\right),
$$

converges weakly to $u$ in $W_{0}^{1,1}(\Omega)$, where $u$ is the solution of a homogenized problem

$$
-\operatorname{div}\left(b(D u)=f \quad \text { on } \Omega, u \in W_{0}^{1, p}(\Omega) .\right.
$$

This paper is organized in the following way: In Section 2 we fix some notation and present necessary preliminary results. Section 3 contains the homogenization result described above, which also is the main result of this paper. In Section 4 we derive a homogenization result for an auxiliary problem. A key ingredient in the proof of the main result is that the solutions of the auxiliary problem are used to define a special type of test function. Finally, in Section 5 we give some properties of the homogenized operator $b$.

\section{$\S 2$. Preliminaries and Notation}

Let $\Omega$ be a regular bounded open subset of $\mathbf{R}^{n}$ and $|E|$ denote the Lebesgue measure of the set $E$ in $\mathbf{R}^{n}$. Moreover let $\langle\cdot, \cdot\rangle$ denote the Euclidean scalar product on $\mathbf{R}^{n}$ and $\chi_{E}$ the characteristic function of the set $E$. Let $p$ be a real constant $1<p<\infty$ and let $q$ be its conjugate exponent, $1 / p+1 / q=1$. We will denote by $C$ and $C_{i}$ constants that may change from one place to another.

Furthermore, let $Y=Z=(0,1)^{n}$ be the unit cube in $\mathbf{R}^{n}$. Let $\left\{\Omega_{i} \subset Y: i=1, \cdots, N\right\}$ be a family of disjoint open sets such that $\left|Y \backslash \bigcup_{i=1}^{N} \Omega_{i}\right|=0$ and $\left|\partial \Omega_{i}\right|=0$.

Let $\lambda$ be a weight on $\mathbf{R}^{n}$, i.e. $\lambda$ is measurable and

$$
\lambda>0 \text { a.e., } \lambda \text { and } \lambda^{-1 /(p-1)} \text { are in } L_{\text {loc }}^{1}\left(\mathbf{R}^{n}\right) \text {. }
$$

We denote by $L^{p}(\Omega, \lambda)$ the set of real functions $u$ in $L_{\text {loc }}^{1}(\Omega)$ such that $u \lambda^{1 / p}$ is in $L^{p}(\Omega)$, by $W^{1, p}(\Omega, \lambda)$ the set of the functions $u$ in $W_{\text {loc }}^{1,1}(\Omega)$ such that $u \in L^{p}(\Omega, \lambda)$ and $D u \in$ $\left[L^{p}(\Omega, \lambda)\right]^{n}$. Moreover, we denote by $W_{0}^{1, p}(\Omega, \lambda)$ the completion of $C_{0}^{1}(\Omega)$ with respect to the norm in $W^{1, p}(\Omega, \lambda)$, i.e.

$$
\|u\|_{W^{1, p}(\Omega, \lambda)}=\left(\int_{\Omega}\left(|u|^{p}+|D u|^{p}\right) \lambda d x\right)^{1 / p} .
$$

By $C_{\text {per }}^{1}(Y)$ we mean the set of all $Y$-periodic functions in $C^{1}\left(\mathbf{R}^{n}\right)$ with mean value zero. We also define $W_{\text {per }}^{1, p}(\Omega, \lambda)$ as the set of real functions $u$ in $W_{\text {loc }}^{1,1}\left(\mathbf{R}^{n}\right)$ with mean value zero such that $u$ is $Y$-periodic and $u \in W^{1, p}(Y, \lambda)$. 
We now define the Muckenhoupt $A_{p}$ class:

Definition 2.1. Let $p>1, K \geq 1$ and let $\lambda$ be a weight on $\mathbf{R}^{n}$. Then $\lambda$ is in the class $A_{p}(K)$ if

$$
\left(\frac{1}{|Q|} \int_{Q} \lambda d z\right)\left(\frac{1}{|Q|} \int_{Q} \lambda^{-\frac{1}{p-1}} d z\right)^{p-1} \leq K
$$

for every cube $Q \in \mathbf{R}^{n}$ with faces parallel to the coordinate planes. We set $A_{p}=\bigcup_{K \geq 1} A_{p}(K)$.

Let $\lambda_{i}$ be a $Y$-periodic weight on $\mathbf{R}^{n}$, i.e. $\lambda_{i}$ satisfies (2.1) and is $Y$-periodic. We define the weights $\bar{\lambda}_{h}$ and $\lambda_{h}$ as

$$
\bar{\lambda}_{h}(x)=\sum_{i=1}^{N} \chi_{\Omega_{i}}(x) \lambda_{i}(h x), \quad \lambda_{h}(x)=\sum_{i=1}^{N} \chi_{\Omega_{i}}(h x) \lambda_{i}\left(h^{2} x\right) .
$$

Then it follows that $\bar{\lambda}_{h}, \bar{\lambda}_{h}{ }^{-1 /(p-1)}, \lambda_{h}$ and $\lambda_{h}{ }^{-1 /(p-1)}$ all are in $L_{\text {loc }}^{1}\left(\mathbf{R}^{n}\right)$. Moreover, we assume that $\lambda_{i}, \bar{\lambda}_{h}$ and $\lambda_{h}$ are in $A_{p}(K)$ for some $K$.

Let $a: \mathbf{R}^{n} \times \mathbf{R}^{n} \times \mathbf{R}^{n} \rightarrow \mathbf{R}^{n}$ be a function such that

$$
a(y, z, \xi)=\sum_{i=1}^{N} \chi_{\Omega_{i}}(y) a_{i}(z, \xi) .
$$

We assume that $a(\cdot, z, \xi)$ is $Y$-periodic and $a(y, \cdot, \xi)$ is $Z$-periodic. We also assume that $a$ satisfies certain continuity and monotonicity conditions. To be more specific, assume that there exist constants $c_{1}^{i}, c_{2}^{i}>0$ and constants $\alpha$ and $\beta$ with $0 \leq \alpha \leq \min (1, p-1)$ and $\max (p, 2) \leq \beta<\infty$ such that

$$
\begin{aligned}
& \left|a_{i}\left(z, \xi_{1}\right)-a_{i}\left(z, \xi_{2}\right)\right| \leq c_{1}^{i} \lambda_{i}(z)\left(1+\left|\xi_{1}\right|+\left|\xi_{2}\right|\right)^{p-1-\alpha}\left|\xi_{1}-\xi_{2}\right|^{\alpha}, \\
& \left\langle a_{i}\left(z, \xi_{1}\right)-a_{i}\left(z, \xi_{2}\right), \xi_{1}-\xi_{2}\right\rangle \geq c_{2}^{i} \lambda_{i}(z)\left(1+\left|\xi_{1}\right|+\left|\xi_{2}\right|\right)^{p-\beta}\left|\xi_{1}-\xi_{2}\right|^{\beta},
\end{aligned}
$$

for a.e. $z \in \mathbf{R}^{n}$, every $\xi \in \mathbf{R}^{n}$. Moreover we assume that

$$
a_{i}(z, 0)=0
$$

for a.e. $z \in \mathbf{R}^{n}$.

As a direct consequence of (2.4), (2.5), and (2.6) the following inequalities hold:

$$
\begin{aligned}
& \left|a_{i}(z, \xi)\right| \leq c_{a}^{i} \lambda_{i}(z)\left(1+|\xi|^{p-1}\right), \\
& \lambda_{i}(z)|\xi|^{p} \leq c_{b}^{i}\left(\lambda_{i}(z)+\left\langle a_{i}(z, \xi), \xi\right\rangle\right), \\
& \int_{Z}\left|\xi+D v_{i}^{\xi}\right|^{p} \lambda_{i}(z) d z \leq c_{c}^{i}\left(1+|\xi|^{p}\right) .
\end{aligned}
$$

In [8] the following result is proved.

Lemma 2.1. Let $p>1$ and $K \geq 1$. Then there exist two positive constants $\delta=\delta(n, p, K)$ and $C=C(n, p, K)$ such that

$$
\begin{gathered}
\left(\frac{1}{|Q|} \int_{Q} \lambda^{1+\delta} d y\right)^{\frac{1}{1+\delta}} \leq C \frac{1}{|Q|} \int_{Q} \lambda d y \\
\left(\frac{1}{|Q|} \int_{Q} \lambda^{-(1+\delta) /(p-1)} d y\right)^{\frac{1}{1+\delta}} \leq C \frac{1}{|Q|} \int_{Q} \lambda^{-1 /(p-1)} d y,
\end{gathered}
$$

for every cube with faces parallel to the coordinate planes and every $\lambda \in A_{p}(K)$.

In [9] the following weighted compensated compactness result is proved.

Lemma 2.2. Let $\nu \in A_{p}, K \geq 1$, let $\left(\lambda_{h}\right)$ be a family in $A_{p}(K)$ and let $\Omega$ be an open bounded set. Let $\left(u_{h}\right)$ be a family of functions satisfying

(1) $\int_{\Omega}\left|D u_{h}\right|^{p} \lambda_{h} d y \leq C_{1}<\infty$ for every $h \in \mathbf{N}$,

(2) there exists a function $u \in W^{1, p}(\Omega, \nu)$ such that $u_{h} \rightarrow u$ in $L^{1}(\Omega)$. 
Moreover, let $\left(a_{h}\right)$ be a family of vector functions in $\mathbf{R}^{n}$ such that

(3) $\int_{\Omega}\left|a_{h}\right|^{q} \lambda_{h}^{-1 /(p-1)} d y \leq C_{2}<\infty$ for every $h \in \mathbf{N}$,

(4) $\operatorname{div}\left(a_{h}\right)=f \in L^{\infty}(\Omega)$ on $C_{0}^{1}(\Omega)$ for every $h \in \mathbf{N}$, Then

(5) there exists $a \in\left[L^{q}\left(\Omega, \nu^{-1 /(p-1)}\right)\right]^{n}$ such that $a_{h} \rightarrow$ a weakly in $\left[L^{1}(\Omega)\right]^{n}$.

$$
\int_{\Omega}\left\langle a_{h}, D u_{h}\right\rangle \phi d y \rightarrow \int_{\Omega}\langle a, D u\rangle \phi d y
$$

for every $\phi \in C_{0}^{\infty}(\Omega)$.

In [15] the following convergence result for periodic functions is proved.

Lemma 2.3. Let $1 \leq p \leq \infty$ and let $u_{h} \in L_{\mathrm{loc}}^{p}\left(\mathbf{R}^{n}\right)$ be $Y$-periodic for $h \in \mathbf{N}$. Moreover, suppose that $u_{h} \rightarrow u$ weakly in $L^{p}(Y)$ (weakly* if $p=\infty$ ) as $h \rightarrow \infty$. Let $w_{h}$ be defined by $w_{h}(x)=u_{h}(h x)$. Then as $h \rightarrow \infty$ it holds that $w_{h} \rightarrow \frac{1}{|Y|} \int_{Y} u(y) d y$ weakly in $L^{p}(\Omega)$ (weakly* if $\left.p=\infty\right)$.

We end this section with a simple extension lemma.

Lemma 2.4. Let $\lambda$ be a $Y$-periodic weight on $\mathbf{R}^{n}$; let $g: Y \rightarrow \mathbf{R}^{n}$ be a function such that

$$
g \in\left[L^{q}\left(Y, \lambda^{-1 /(p-1)}\right]^{n}, \quad \int_{Y}\langle g, D w\rangle d y=0, \forall w \in W_{\mathrm{per}}^{1, p}(Y, \lambda),\right.
$$

and let $\widetilde{g}$ be the $Y$-periodic extension to $\mathbf{R}^{n}$ of $g$. Then we have

$$
\widetilde{g} \in\left[L_{\mathrm{loc}}^{q}\left(\mathbf{R}^{n}, \lambda^{-1 /(p-1)}\right)\right]^{n}, \quad \int_{\mathbf{R}^{n}}\langle\widetilde{g}, D v\rangle d y=0, \forall v \in C_{0}^{1}\left(\mathbf{R}^{n}\right) .
$$

In situations where no confusion can occur we will use the same notation for the extended function as for the original one.

\section{$\S 3$. The Main Theorem}

Let us consider the following Dirichlet problems:

$$
\left\{\begin{array}{l}
\int_{\Omega}\left\langle a\left(h x, h^{2} x, D u_{h}\right), D \phi\right\rangle d x=\int_{\Omega} f \phi d x, \forall \phi \in W_{0}^{1, p}\left(\Omega, \lambda_{h}\right), \\
u_{h} \in W_{0}^{1, p}\left(\Omega, \lambda_{h}\right)
\end{array}\right.
$$

where $f \in L^{\infty}(\Omega)$. By standard results in existence theory there exist unique solutions for each $h$. Below we state the main result of this paper.

Theorem 3.1. Let $\left(u_{h}\right)$ be the solutions of (3.1). Then

$$
\begin{aligned}
u_{h} & \rightarrow u \text { weakly in } W_{0}^{1,1}(\Omega), \\
a\left(h x, h^{2} x, D u_{h}\right) & \rightarrow b(D u) \text { weakly in }\left[L^{1}(\Omega)\right]^{n},
\end{aligned}
$$

as $h \rightarrow \infty$, where $u$ is the unique solution of

$$
\left\{\begin{array}{l}
\int_{\Omega}\langle b(D u), D \phi\rangle d x=\int_{\Omega} f \phi d x \text { for every } \phi \in W_{0}^{1, p}(\Omega), \\
u \in W_{0}^{1, p}(\Omega) .
\end{array}\right.
$$

The operator $b: \mathbf{R}^{n} \rightarrow \mathbf{R}^{n}$ is defined as

$$
b(\xi)=\frac{1}{|Y|} \int_{Y} b_{Y}\left(y, \xi+D u^{\xi}(y)\right) d y,
$$

where $u^{\xi}$ is the unique solution of the $Y$-cell problem

$$
\left\{\begin{array}{l}
\int_{Y}\left\langle b_{Y}\left(y, \xi+D u^{\xi}(y)\right), D \phi\right\rangle d y=0 \text { for every } \phi \in W_{\mathrm{per}}^{1, p}(Y), \\
u^{\xi} \in W_{\mathrm{per}}^{1, p}(Y) .
\end{array}\right.
$$

The operator $b_{Y}: Y \times \mathbf{R}^{n} \rightarrow \mathbf{R}^{n}$ is defined as $b_{Y}(y, \xi)=\sum_{i=1}^{N} \chi_{\Omega_{i}}(y) b_{i}(\xi)$, where

$$
b_{i}(\xi)=\frac{1}{|Z|} \int_{Z} a_{i}\left(z, \xi+D v_{i}^{\xi}(z)\right) d z
$$


and $v_{i}^{\xi}$ are the unique solutions of the $Z$-cell problems

$$
\left\{\begin{array}{l}
\int_{Z}\left\langle a_{i}\left(z, \xi+D v_{i}^{\xi}(z)\right), D \phi(z)\right\rangle d z=0 \text { for every } \phi \in W_{\text {per }}^{1, p}\left(Z, \lambda_{i}\right) \\
v_{i}^{\xi} \in W_{\text {per }}^{1, p}\left(Z, \lambda_{i}\right) .
\end{array}\right.
$$

Proof. Let us first prove that $\left\|D u_{h}\right\|_{L^{p}\left(\Omega, \lambda_{h}\right)} \leq C$. By periodicity it follows that

$$
\int_{\Omega} \lambda_{h}(x) d x \leq C, \quad \int_{\Omega}\left(\lambda_{h}(x)\right)^{-1 /(p-1)} d x \leq C .
$$

Thus, by (2.8), (3.1), (3.3), Poincare's and Hölder's inequalities we obtain

$$
\begin{aligned}
& \int_{\Omega}\left|D u_{h}\right|^{p} \lambda_{h}(x) d x \leq c_{b}\left(\int_{\Omega} \lambda_{h}(x) d x+\int_{\Omega} f u_{h} d x\right) \\
\leq & C\left(\int_{\Omega} \lambda_{h}(x) d x+\|f\|_{L^{\infty}(\Omega)} \int_{\Omega}\left|D u_{h}\right| d x\right) \leq C\left(1+\left(\int_{\Omega}\left|D u_{h}\right|^{p} \lambda_{h}(x) d x\right)^{1 / p}\right) .
\end{aligned}
$$

Hence it is clear that

$$
\left\|D u_{h}\right\|_{L^{p}\left(\Omega, \lambda_{h}\right)} \leq C \text {. }
$$

Let $\eta_{h}$ be defined as $\eta_{h}=a\left(h x, h^{2} x, D u_{h}\right)$. Then (2.7) and (3.4) implies

$$
\left\|\eta_{h}\right\|_{\left[L^{q}\left(\Omega, \lambda_{h}^{-1 /(p-1)}\right)\right]^{n}} \leq C \text {. }
$$

Take $\delta>0$ such that (2.11) holds. Now choose $\sigma_{1}$ such that

$$
\frac{1+\sigma_{1}}{p-1-\sigma_{1}}=\frac{1+\delta}{p-1} \text {. }
$$

Then $\sigma_{1}>0$ and $p-1-\sigma_{1}>0$. Let $Q$ be a cube in $\mathbf{R}^{n}$ containing $\Omega$. Hölder's inequality and (3.4) then gives

$$
\begin{aligned}
\int_{\Omega}\left|D u_{h}\right|^{1+\sigma_{1}} d x & \leq\left(\int_{\Omega}\left|D u_{h}\right|^{p} \lambda_{h} d x\right)^{\frac{1+\sigma_{1}}{p}}\left(\int_{\Omega} \lambda_{h}^{-\frac{1+\sigma_{1}}{p-1-\sigma_{1}}} d x\right)^{\frac{p-1-\sigma_{1}}{p}} \\
& \leq C\left(\int_{\Omega} \lambda_{h}^{-\frac{1+\delta}{p-1}} d x\right)^{\frac{p-1}{p+\delta}} \leq C\left(\int_{Q} \lambda_{h}^{-\frac{1+\delta}{p-1}} d x\right)^{\frac{p-1}{p+\delta}}
\end{aligned}
$$

By applying (2.11) and (3.3) in the inequality above, we obtain

$$
\int_{\Omega}\left|D u_{h}\right|^{1+\sigma_{1}} d x \leq C\left(\int_{Q} \lambda_{h}{ }^{1 /(1-p)} d x\right)^{(p-1)(1+\delta) /(p+\delta)} \leq C .
$$

Next, choose $\delta>0$ such that (2.10) holds and choose $\sigma_{2}$ such that

$$
1+\delta=\left(1+\sigma_{2}\right) \frac{q-1}{q-1-\sigma_{2}} .
$$

Then $\sigma_{2}>0$ and $q-1-\sigma_{2}>0$. By using (2.10) and arguing similarly as for (3.6) we obtain

$$
\int_{\Omega}\left|\eta_{h}\right|^{1+\sigma_{2}} d x \leq C
$$

This means that $\left(u_{h}\right)$ and $\left(\eta_{h}\right)$ are bounded in $W_{0}^{1,1+\sigma_{1}}(\Omega)$ and $\left[L^{1+\sigma_{2}}(\Omega)\right]^{n}$ respectively. Since these spaces are reflexive, we have that there exist subsequences, still denoted by $\left(u_{h}\right)$ and $\left(\eta_{h}\right)$, such that

$$
\begin{aligned}
& u_{h} \rightarrow u_{*} \text { weakly in } W_{0}^{1,1+\sigma_{1}}(\Omega), \\
& \eta_{h} \rightarrow \eta_{*} \text { weakly in }\left[L^{1+\sigma_{2}}(\Omega)\right]^{n} .
\end{aligned}
$$

From (3.7) and (3.8) it follows that

$$
\begin{aligned}
& u_{h} \rightarrow u_{*} \text { weakly in } W_{0}^{1,1}(\Omega), \\
& \eta_{h} \rightarrow \eta_{*} \text { weakly in }\left[L^{1}(\Omega)\right]^{n} .
\end{aligned}
$$


From our original problem (3.1) we have

$$
\int_{\Omega}\left\langle\eta_{h}, D \phi\right\rangle d x=\int_{\Omega} f \phi d x \text { for every } \phi \in W_{0}^{1, p}\left(\Omega, \lambda_{h}\right) .
$$

By using the fact $C_{0}^{1}(\Omega) \subset W_{0}^{1, p}\left(\Omega, \lambda_{h}\right)$ and (3.10) we can pass to the limit in (3.11), thus

$$
\int_{\Omega}\left\langle\eta_{*}, D \phi\right\rangle d x=\int_{\Omega} f \phi d x \text { for every } \phi \in C_{0}^{1}(\Omega) .
$$

Density and the fact that $\eta_{*} \in\left[L^{q}(\Omega)\right]^{n}$ (see (3.17)) then gives

$$
\int_{\Omega}\left\langle\eta_{*}, D \phi\right\rangle d x=\int_{\Omega} f \phi d x \text { for every } \phi \in W_{0}^{1, p}(\Omega) .
$$

Let us now observe that by (3.9), (3.10) and (3.12) the theorem is proved if we show that

$$
\begin{aligned}
& u_{*} \in W_{0}^{1, p}(\Omega), \\
& \eta_{*}=b\left(D u_{*}\right) \text { a.e. on } \Omega,
\end{aligned}
$$

since the uniqueness of the solution of the homogenized problem (3.2) then implies that $u_{*}=u$ a.e. on $\Omega$.

We start with the proof of (3.13). We observe that since $\Omega$ is a regular bounded open set it is sufficient to show that $D u_{*} \in\left[L^{p}(\Omega)\right]^{n}$. Let $\phi \in C_{0}(\Omega)$. Then Hölder's inequality and (3.4) gives

$$
\begin{aligned}
\left\|D u_{h} \phi\right\|_{\left[L^{1}(\Omega)\right]^{n}} & \leq\left(\int_{\Omega}\left|D u_{h}\right|^{p} \lambda_{h}(x) d x\right)^{\frac{1}{p}}\left(\int_{\Omega}\left(\lambda_{h}(x)\right)^{-\frac{1}{p-1}}|\phi|^{q} d x\right)^{\frac{1}{q}} \\
& \leq C\left(\int_{\Omega}\left(\lambda_{h}(x)\right)^{-\frac{1}{p-1}}|\phi|^{q} d x\right)^{\frac{1}{q}} .
\end{aligned}
$$

Applying lim inf on both sides of (3.15) and using the weak lower semicontinuity of the norm on the left hand side and periodicity on the right hand side we obtain

$$
\int_{\Omega}\left|D u_{*}\right||\phi| d x \leq C\|\phi\|_{L^{q}(\Omega)} \text { for every } \phi \in C_{0}(\Omega) .
$$

By density and Landau's theorem we then have that

$$
D u_{*} \in\left[L^{p}(\Omega)\right]^{n} .
$$

By using (3.5) and arguments similar to those employed in the proof of (3.16) it can also be deduced that

$$
\eta_{*} \in\left[L^{q}(\Omega)\right]^{n} .
$$

It remains to prove (3.14). For this purpose let us define the test function

$$
w_{h}^{\xi}(x)=(\xi, x)+\frac{1}{h} u_{h}^{\xi}(h x),
$$

where $u_{h}^{\xi}$ is defined as in the auxiliary problem (see Section 4). To be able to apply the compensated compactness result (Lemma 2.2) we have to prove certain facts about $w_{h}^{\xi}$ and $a\left(h x, h^{2} x, D w_{h}^{\xi}\right)$. Therefore, by periodicity, (4.5) and the fact that $\lambda_{h}(x)=\bar{\lambda}_{h}(h x)$ we get

$$
\int_{\Omega}\left|D w_{h}^{\xi}\right|^{p} \lambda_{h}(x) d x \leq C .
$$

Moreover, by using (2.7) and (3.18) we obtain

$$
\int_{\Omega}\left|a\left(h x, h^{2} x, D w_{h}^{\xi}\right)\right|^{q}\left(\lambda_{h}(x)\right)^{-1 /(p-1)} d x \leq C .
$$


By periodicity and Lemma 2.3 we have that

$$
\begin{aligned}
w_{h}^{\xi}(\cdot) & \rightarrow(\xi, \cdot) \text { in } L^{1}(\Omega), \\
a\left(h x, h^{2} x, D w_{h}^{\xi}\right) & \rightarrow \frac{1}{|Y|} \int_{Y} b_{Y}\left(y, \xi+D u^{\xi}\right) d y=b(\xi) \text { weakly in }\left[L^{1}(\Omega)\right]^{n} .
\end{aligned}
$$

Finally, due to (4.6), we can apply Lemma 2.4 on (4.1) and obtain

$$
\operatorname{div}\left(a\left(h x, h^{2} x, D w_{h}^{\xi}\right)\right)=0 \text { on } C_{0}^{1}(\Omega),
$$

and we are now ready to apply the compensated compactness result. Indeed, by the monotonicity of $a$ we have for a fixed $\xi$ that

$$
\int_{\Omega}\left\langle\eta_{h}-a\left(h x, h^{2} x, D w_{h}^{\xi}(x)\right), D u_{h}(x)-D w_{h}^{\xi}(x)\right\rangle \phi(x) d x \geq 0
$$

for every $\phi \in C_{0}^{\infty}(\Omega), \phi \geq 0$. By the compensated compactness lemma (Lemma 2.2) with the weight $\nu=1$, we get in the limit

$$
\int_{\Omega}\left\langle\eta_{*}-b(\xi), D u_{*}(x)-\xi\right\rangle \phi(x) d x \geq 0
$$

for every $\phi \in C_{0}^{\infty}(\Omega), \phi \geq 0$. Hence for our fixed $\xi \in \mathbf{R}^{n}$ we have that

$$
\left\langle\eta_{*}-b(\xi), D u_{*}(x)-\xi\right\rangle \geq 0 \text { for a.e. } x \in \Omega \text {. }
$$

By density and the continuity of $b$ (see Lemma 5.2), it follows that

$$
\left\langle\eta_{*}-b(\xi), D u_{*}(x)-\xi\right\rangle \geq 0 \text { for a.e. } x \in \Omega \text { and every } \xi \in \mathbf{R}^{n} \text {. }
$$

Since $b$ is monotone (5.3) and continuous (5.4), we have that $b$ is maximal monotone and hence (3.14) follows. Finally, let us observe that we have proved the theorem only up to a subsequence, but since the homogenized operator is uniquely defined and the solution of the homogenized problem is unique we can conclude that the theorem holds for the whole sequence.

\section{§4. An Auxiliary Problem}

In this section we prove a homogenization result for the auxiliary problem. This result was used in the definition of the special type of test functions defined in the proof of the main result (Theorem 3.1) of this paper.

Fix $\xi$ and consider the following Dirichlet problems:

$$
\left\{\begin{array}{l}
\int_{Y}\left\langle a\left(y, h y, \xi+D u_{h}^{\xi}\right), D \phi\right\rangle d y=0, \forall \phi \in W_{\text {per }}^{1, p}\left(Y, \bar{\lambda}_{h}\right) \\
u_{h}^{\xi} \in W_{\text {per }}^{1, p}\left(Y, \bar{\lambda}_{h}\right)
\end{array}\right.
$$

By standard results in existence theory there exist unique solutions for each $h$. Below we state the auxiliary result of this paper.

Theorem 4.1. Let $\left(u_{h}^{\xi}\right)$ be the solutions of (4.1). We then have that

$$
\begin{aligned}
u_{h}^{\xi} & \rightarrow u^{\xi} \text { weakly in } W_{\text {per }}^{1,1}(Y), \\
a\left(y, h y, \xi+D u_{h}^{\xi}\right) & \rightarrow b_{Y}\left(y, \xi+D u^{\xi}\right) \text { weakly in }\left[L^{1}(Y)\right]^{n},
\end{aligned}
$$

as $h \rightarrow \infty$, where $u^{\xi}$ is the unique solution of

$$
\left\{\begin{array}{l}
\int_{Y}\left\langle b_{Y}\left(y, \xi+D u^{\xi}\right), D \phi\right\rangle d y=0 \text { for every } \phi \in W_{\text {per }}^{1, p}(Y), \\
u^{\xi} \in W_{\text {per }}^{1, p}(Y)
\end{array}\right.
$$

The operator $b_{Y}: Y \times \mathbf{R}^{n} \rightarrow \mathbf{R}^{n}$ is defined as $b_{Y}(y, \tau)=\sum_{i=1}^{N} \chi_{\Omega_{i}}(y) b_{i}(\tau)$, where

$$
b_{i}(\tau)=\frac{1}{|Z|} \int_{Z} a_{i}\left(z, \tau+D v_{i}^{\tau}(z)\right) d z
$$


and $v_{i}^{\tau}$ are the unique solutions of the $Z$-cell problems

$$
\left\{\begin{array}{l}
\int_{Z}\left\langle a_{i}\left(z, \tau+D v_{i}^{\tau}(z)\right), D \phi(z)\right\rangle d z=0, \forall \phi \in W_{\text {per }}^{1, p}\left(Z, \lambda_{i}\right) \\
v_{i}^{\tau} \in W_{\text {per }}^{1, p}\left(Z, \lambda_{i}\right)
\end{array}\right.
$$

Proof. By (2.8), (4.1) and (2.7) we have that

$$
\int_{Y}\left|\xi+D u_{h}^{\xi}\right|^{p} \bar{\lambda}_{h}(y) d y \leq C_{b}\left(\int_{Y} \bar{\lambda}_{h}(y) d y+C_{a} \int_{Y}\left(1+\left|\xi+D u_{h}^{\xi}\right|^{p-1}\right) \bar{\lambda}_{h}(y)|\xi| d y\right) .
$$

Moreover, if we use Young's inequality on the right hand side and rearrange the resulting inequality we obtain

$$
\begin{aligned}
& \left(1-\frac{C_{a} C_{b} 2^{q-1} \eta}{q}\right) \int_{Y}\left|\xi+D u_{h}^{\xi}\right|^{p} \bar{\lambda}_{h}(y) d y \\
\leq & C_{b}\left(1+\frac{C_{a} 2^{q-1} \eta}{q}+\frac{C_{a}|\xi|^{p} \eta^{-(p-1)}}{p}\right) \int_{Y} \bar{\lambda}_{h}(y) d y,
\end{aligned}
$$

where $\eta$ is a positive real number. By choosing $\eta$ small enough we get that

$$
\int_{Y}\left|\xi+D u_{h}^{\xi}\right|^{p} \bar{\lambda}_{h}(y) d y \leq C .
$$

In particular this implies

$$
\int_{Y}\left|D u_{h}^{\xi}\right|^{p} \bar{\lambda}_{h}(y) d y \leq C .
$$

Let us define $\eta_{h}^{i}=a_{i}\left(h y, \xi+D u_{h}^{\xi}\right)$. By using (2.7) and (4.4), it follows that

$$
\int_{\Omega_{i}}\left|\eta_{h}^{i}\right|^{q}\left(\lambda_{i}(h y)\right)^{-1 /(p-1)} d y \leq C .
$$

Moreover, by using (2.11), (4.5), (2.10), (4.6) and arguments similar to those employed in the proof of (3.6), it can be deduced that

$$
\int_{Y}\left|D u_{h}^{\xi}\right|^{1+\kappa_{1}} d y \leq C, \quad \int_{\Omega_{i}}\left|\eta_{h}^{i}\right|^{1+\kappa_{2}} d y \leq C .
$$

Thus we have that $\left(u_{h}^{\xi}\right)$ and $\left(\eta_{h}^{i}\right)$ are bounded in $W_{\text {per }}^{1,1+\kappa_{1}}(Y)$ and $\left[L^{1+\kappa_{2}}\left(\Omega_{i}\right)\right]^{n}$ respectively. Since these spaces are reflexive, there exist subsequences, still denoted by $\left(u_{h}^{\xi}\right)$ and $\left(\eta_{h}^{i}\right)$, such that

$$
u_{h}^{\xi} \rightarrow u_{*}^{\xi} \text { weakly in } W_{\text {per }}^{1,1+\kappa_{1}}(Y), \quad \eta_{h}^{i} \rightarrow \eta_{*}^{i} \text { weakly in }\left[L^{1+\kappa_{2}}\left(\Omega_{i}\right)\right]^{n} .
$$

Hence we can conclude that

$$
u_{h}^{\xi} \rightarrow u_{*}^{\xi} \text { weakly in } W_{\text {per }}^{1,1}(Y), \quad \eta_{h}^{i} \rightarrow \eta_{*}^{i} \text { weakly in }\left[L^{1}\left(\Omega_{i}\right)\right]^{n} .
$$

Using similar ideas as in the proof of (3.16), it can be shown that

$$
\eta_{*}^{i} \in\left[L^{q}(Y)\right]^{n} .
$$

From (4.1) and (4.7) it follows that

$$
\sum_{i=1}^{N} \int_{\Omega_{i}}\left\langle\eta_{*}^{i}, D \phi\right\rangle d y=0 \text { for every } \phi \in C_{\mathrm{per}}^{1}(Y) .
$$

Density arguments in conjunction with (4.8) then results in

$$
\sum_{i=1}^{N} \int_{\Omega_{i}}\left\langle\eta_{*}^{i}, D \phi\right\rangle d y=0 \text { for every } \phi \in W_{\text {per }}^{1, p}(Y) .
$$


Thus the theorem is proved if we show that

$$
\begin{aligned}
& u_{*}^{\xi} \in W_{\mathrm{per}}^{1, p}(Y), \\
& \eta_{*}^{i}=b_{i}\left(\xi+D u_{*}^{\xi}\right) \text { a.e. on } \Omega_{i},
\end{aligned}
$$

since the uniqueness of the solution of the homogenized problem (4.2) then implies that $u_{*}^{\xi}=u^{\xi}$ a.e. on $Y$.

Let us start with (4.9). We observe that since $Y$ is a regular bounded open set it is sufficient to show that $D u_{*}^{\xi} \in\left[L^{p}(Y)\right]^{n}$ which is obtained using the same ideas as in the proof of (3.16).

It remains to prove (4.10). Therefore let us define the test function $w_{h}^{\tau, i}$ by

$$
w_{h}^{\tau, i}(y)=(\tau, y)+\frac{1}{h} v_{i}^{\tau}(h y),
$$

where $v_{i}^{\tau} \in W_{\text {per }}^{1, p}\left(Z, \lambda_{i}\right)$ is defined as in (4.3). To be able to apply the compensated compactness result (Lemma 2.2) we have to prove certain facts about $w_{h}^{\tau, i}$ and $a_{i}\left(h y, D w_{h}^{\tau, i}\right)$. Indeed,

$$
\int_{\Omega_{i}}\left|D w_{h}^{\tau, i}\right|^{p} \lambda_{i}(h y) d y \leq C
$$

follows from (4.11). Moreover, we also have that

$$
\int_{\Omega_{i}}\left|a_{i}\left(h y, D w_{h}^{\tau, i}\right)\right|^{q}\left(\lambda_{i}(h y)\right)^{-1 /(p-1)} d y \leq C
$$

by (2.7) and (4.12). By periodicity we obtain

$$
\begin{aligned}
w_{h}^{\tau, i}(\cdot) & \rightarrow(\tau, \cdot) \text { strongly in } L^{1}\left(\Omega_{i}\right) \\
a_{i}\left(h y, D w_{h}^{\tau, i}\right) & \rightarrow \frac{1}{|Z|} \int_{Z} a_{i}\left(z, \tau+D v_{i}^{\tau}(z)\right) d z=b_{i}(\tau) \text { weakly in }\left[L^{1}\left(\Omega_{i}\right)\right]^{n} .
\end{aligned}
$$

Application of Lemma 2.4 on (4.3) gives $\operatorname{div}\left(a_{i}\left(h y, D w_{h}^{\tau, i}\right)\right)=0$ on $C_{0}^{1}\left(\Omega_{i}\right)$. By the monotonicity of $a_{i}$ we have for a fixed $\tau$ that

$$
\int_{\Omega_{i}}\left\langle\eta_{*}^{i}-a_{i}\left(h y, D w_{h}^{\tau, i}(y)\right), \xi+D u_{h}^{\xi}(y)-D w_{h}^{\tau, i}(y)\right\rangle \phi(y) d y \geq 0
$$

for every $\phi \in C_{0}^{\infty}\left(\Omega_{i}\right), \phi \geq 0$. By the compensated compactness lemma (Lemma 2.2) with $\nu=1$, we then get in the limit

$$
\int_{\Omega_{i}}\left\langle\eta_{*}^{i}-b_{i}(\tau), \xi+D u_{*}^{\xi}(y)-\tau\right\rangle \phi(y) d y \geq 0
$$

for every $\phi \in C_{0}^{\infty}\left(\Omega_{i}\right), \phi \geq 0$. Hence for our fixed $\tau \in \mathbf{R}^{n}$ we have that

$$
\left\langle\eta_{*}^{i}-b_{i}(\tau), \xi+D u_{*}^{\xi}(y)-\tau\right\rangle \geq 0 \text { for a.e. } y \in \Omega_{i}
$$

By density and the continuity of $b_{i}(5.2)$, it follows that

$$
\left\langle a_{*}-b_{i}(\tau), \xi+D u_{*}^{\xi}(y)-\tau\right\rangle \geq 0 \text { for a.e. } y \in \Omega_{i} \text { and every } \tau \in \mathbf{R}^{n} \text {. }
$$

Since $b_{i}$ is monotone $(5.1)$ and continuous $(5.2)$, we have that $b_{i}$ is maximal monotone and hence (4.10) follows.

\section{$\S 5$. Properties of the Homogenized Operators $b_{i}$ and $b$}

In this section we list some properties of the homogenized operators $b_{i}$ and $b$. In particular these properties imply the existence and uniqueness of the solution of the homogenized problem (in the auxiliary and main problem respectively).

Lemma 5.1. Let $b_{i}$ be the homogenized operator defined in Theorem 4.1. Then 
(a) $b_{i}(\cdot)$ is strictly monotone. In particular, we have that

$$
\left\langle b_{i}\left(\tau_{1}\right)-b_{i}\left(\tau_{2}\right), \tau_{1}-\tau_{2}\right\rangle \geq \widetilde{c}_{2}\left(1+\left|\tau_{1}\right|+\left|\tau_{2}\right|\right)^{p-\beta}\left|\tau_{1}-\tau_{2}\right|^{\beta}
$$

for every $\tau_{1}, \tau_{2} \in \mathbf{R}^{n}$.

(b) $b_{i}(\cdot)$ is continuous. In particular, we have for $\gamma=\frac{\alpha}{\beta-\alpha}$ that

$$
\left|b_{i}\left(\tau_{1}\right)-b_{i}\left(\tau_{2}\right)\right| \leq \widetilde{c}_{1}\left(1+\left|\tau_{1}\right|+\left|\tau_{2}\right|\right)^{p-1-\gamma}\left|\tau_{1}-\tau_{2}\right|^{\gamma}
$$

for every $\tau_{1}, \tau_{2} \in \mathbf{R}^{n}$.

(c) $b_{i}(0)=0$.

Proof. These properties follow by using the same ideas as in [3] and [4].

Lemma 5.2. Let $b$ be the homogenized operator defined in Theorem 3.1. Then

(a) $b(\cdot)$ is strictly monotone. In particular, we have that

$$
\left\langle b\left(\xi_{1}\right)-b\left(\xi_{2}\right), \xi_{1}-\xi_{2}\right\rangle \geq \widetilde{C}_{2}\left(1+\left|\xi_{1}\right|+\left|\xi_{2}\right|\right)^{p-\beta}\left|\xi_{1}-\xi_{2}\right|^{\beta}
$$

for every $\xi_{1}, \xi_{2} \in \mathbf{R}^{n}$.

(b) $b(\cdot)$ is continuous. In particular, we have for $\delta=\frac{\gamma}{\beta-\gamma}=\frac{\alpha}{(\beta-\alpha) \beta-\alpha}$ that

$$
\left|b\left(\xi_{1}\right)-b\left(\xi_{2}\right)\right| \leq \widetilde{C}_{1}\left(1+\left|\xi_{1}\right|+\left|\xi_{2}\right|\right)^{p-1-\delta}\left|\xi_{1}-\xi_{2}\right|^{\delta}
$$

for every $\xi_{1}, \xi_{2} \in \mathbf{R}^{n}$.

(c) $b(0)=0$.

Proof. These properties follow by using (5.1) and (5.2) in the corresponding theorem given in for example [3] and [4].

\section{REFERENCES}

[1] Bensoussan, A., Lions, J. L. \& Papanicolaou, G., Asymptotic analysis for periodic structures [M], North Holland, Amsterdam, 1978.

[2] Braides, A. \& Defranceschi, A., Homogenization of multiple integrals [M], Oxford University Press, New York, 1998.

[3] Byström, J., Correctors for some nonlinear monotone operators [R], Research report, No. 11, ISSN: 1400-4003, Department of Mathematics, Luleå University of Technology, 1999.

[4] Byström, J., Correctors for some nonlinear monotone operators [J], J. Nonlinear Math. Phys., 8:1(2001), $8-30$.

[5] Cherkaev, A. \& Kohn, R., Topics in the mathematical modelling of composite materials [M], Birkhäuser, Boston, 1997.

[6] Chiado Piat, V. \& Defranceschi, A., Homogenization of monotone operators [J], Nonlinear Anal., 14:9(1990), 717-732.

[7] Cioranescu, D. \& Donato, P., An introduction to homogenization [M], Oxford University Press, Oxford, 1999.

[ 8 ] Coifman, R. R. \& Fefferman, C., Weighted norm inequalities for maximal function and singular integrals [J], Studia Math., 51(1974), 241-250.

[9] De Arcangelis, R. \& Serra Cassano, F., On the homogenization of degenerate elliptic equations in divergence form [J], J. Math. Pures Appl., 71(1992), 119-138.

[10] Defranceschi, A., An introduction to homogenization and $G$-convergence [M], Lecture notes, School on homogenization, ICTP, Trieste, 1993.

[11] Fusco, N. \& Moscariello, G., On the homogenization of quasilinear divergence structure operators [J], Annali Mat. Pura Appl., 146(1987), 1-13.

[12] Jikov, V., Kozlov, S. \& Oleinik, O., Homogenization of differential operators and integral functionals [M], Springer-Verlag, Berlin-Heidelberg-New York, 1994.

[13] Lions, J. -L., Lukkassen, D., Persson, L. -E. \& Wall, P., Reiterated homogenization of monotone operators [J], C. R. Acad. Sci. Paris Series I, 330:8(2000), 675-680.

[14] Lions, J. -L., Lukkassen, D., Persson, L. -E. \& Wall, P., Reiterated homogenization of nonlinear monotone operators [J], Chin. Ann. Math., 22B:1(2001), 1-12.

[15] Lukkassen, D. \& Wall, P., On weak convergence of locally periodic functions [J], J. Nonlinear. Math. Phys., 9:1(2002), 42-57 2002. 